\title{
UNA ESPECIE NUEVA DE ARISTOLOCHIA L., SUBSECCIÓN PENTANDRAE (ARISTOLOCHIACEAE) DE LA RESERVA DE LA BIOSFERA SIERRA DE MANANTLÁN, JALISCO, MÉXICO
}

\author{
Francisco J. Santana-MicheL \\ Universidad de Guadalajara, Centro Universitario de la Costa Sur, Departamento \\ de Ecología y Recursos Naturales, Independencia Nacional núm. 151, \\ Apdo. postal 64, 48900 Autlán, Jalisco, México \\ fsantanam@cucsur.udg.mx
}

\section{RESUMEN}

Se describe como especie nueva a Aristolochia manantlanensis Santana-Michel, perteneciente a la subsección Pentandrae Duchr., de la localidad de San Miguel, en la reserva de la biosfera Sierra de Manantlán. Esta especie comparte el hábitat con Zea diploperennis y por sus caracteres morfológicos se relaciona con A. acontophylla Pfeifer, A. karwinskii Duchr. y A. variifolia Duchr.

Palabras clave: Aristolochia manantlanensis, Aristolochiaceae, Jalisco, México, Reserva de la Biosfera Sierra de Manantlán.

\section{ABSTRACT}

Aristolochia manantlanensis Santana-Michel is described as new species in subsecction Pentandrae Duchr., from San Miguel in the Sierra de Manantlán Biosphere Reserve. This species grows with Zea diploperennis Iltis, Doebley \& Guzmán, and by morphological characters it is related to A. acontophylla Pfeifer, A. karwinskii Duchr. and A. variifolia Duchr.

Key words: Aristolochia manantlanensis, Aristolochiaceae, Biosphere Reserve Sierra de Manantlán, Jalisco, Mexico.

*Estudiante de postgrado de la Facultad de Ciencias Biológicas y Agropecuarias, Universidad de Colima. 
La reserva de la biosfera Sierra de Manantlán es importante no sólo por la presencia de Zea diploperennis Iltis, Doebley \& Guzmán, maíz silvestre perenne, endémico (Iltis et al., 1979), sino además por la gran riqueza florística existente en esta región. Vázquez et al. (1995) informaron que en esta área de aproximadamente 140,000 hectáreas existen 2,774 especies de plantas vasculares, cifra que a la luz de conocimientos actuales se acerca a las 3,000 especies, de las cuales cerca de 50 son endémicas.

Exploraciones botánicas recientes en la Sierra de Manantlán han puesto de manifiesto que todavía hay una gran cantidad de plantas por descubrir para la región y de algunas aún se desconoce su ubicación taxonómica.

Para el caso de la familia Aristolochiaceae en el área de la reserva de la biosfera Sierra de Manantlán se han registrado ocho especies: Aristolochia malacophylla Stand1., A. odoratissima L., A. styloglossa Pfeifer y A. taliscana Hook. \& Arn., pertenecientes a la subsección Hexandrae (Pfeifer, 1966) y Aristolochia buntingii Pfeifer, A. foetida H.B.K., A. tequilana S. Watson (Vázquez et al., 1995) y A. luzmariana Santana-Michel, descrita de la Sierra de Manantlán y la Sierra del Halo en Tecalitlán (Santana-Michel, 1995) de la subsección Pentandrae (Pfeifer, 1970).

El taxon que a continuación se describe pertenece a la subsección Pentandrae de Aristolochia y crece en el mismo hábitat que Zea diploperennis Iltis Doebley \& Guzmán.

Aristolochia manantlanensis Santana-Michel sp. nov. Figs. 1 y 2.

Herbae perennes procumbentes, 1-1.5 m longae; folia petiolata, sagittata, attenuata, acuminata, ad basim bilobata, lobis subquadratis rotundatis; flores axillares solitarii, pedunculus bracteolatus, calycis pars basalis partem apicalem adpressa, atropurpurea, limbus ovatus, peltatus ad anthesim, 2.4-2.8 cm longus, $1.7-2.4 \mathrm{~cm}$ latus, purpureus, piloso-fimbriatus trichomatibus $0.7-1 \mathrm{~mm}$ longis, fauce flavoviridi; utriculus oblongo-ellipsoideus, 9-11 mm longus, 5-6 mm diametro, syrinx excentrica, tubulosa 2-2.5 $\mathrm{mm}$ longa, $2 \mathrm{~mm}$ diametro.

Hierbas perennes, procumbentes, de 1-1.5 m de largo; raíz principal axonomorfa; hojas pecioladas, sagitiformes, ápice agudo a acuminado, base bilobada, lóbulos subcuadrados, redondeados, haz y envés glabrescentes, con tricomas diseminados en las nervaduras principales y el borde, nervación actinódroma basal; pecíolo de (1.2) 2-5 (6) cm de largo, 1-1.5 mm de grueso; lámina de (3.6) 5-10 (12) cm de largo, (2.8) 3-5.5 (6) cm de ancho; flores axilares, solitarias, pedúnculo bracteolado, de (3) 


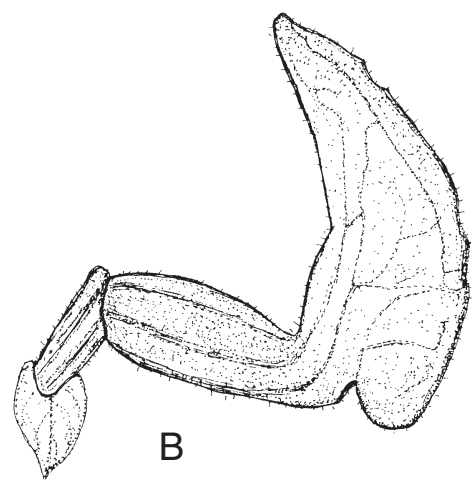

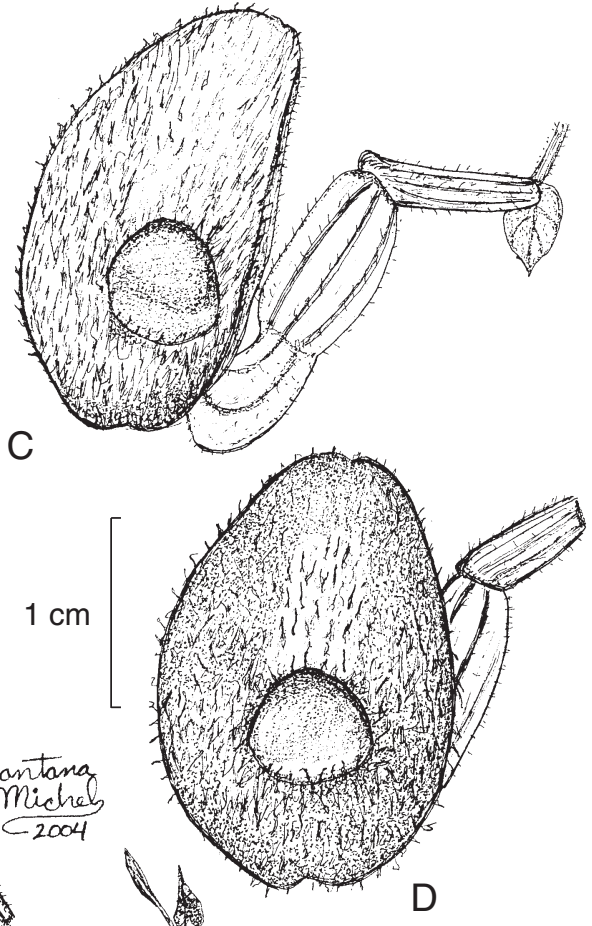
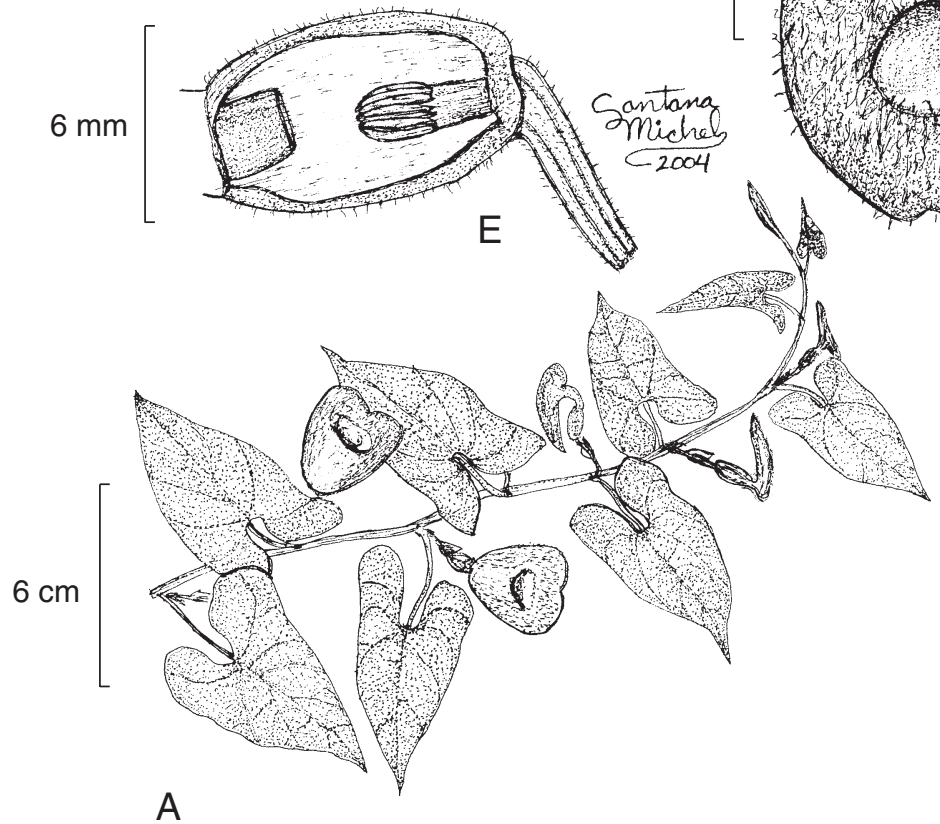

Fig. 1. Aristolochia manantlanensis. A. aspecto de la planta; B. flor cerrada; C. flor vista de costado; D. flor vista de frente; E. corte longitudinal del utrículo, mostrando la siringe y el ginostemo. Ilustación basada en la colección tipo (F. J. Santana-Michel y D. Zizumbo Villarreal 11593). 


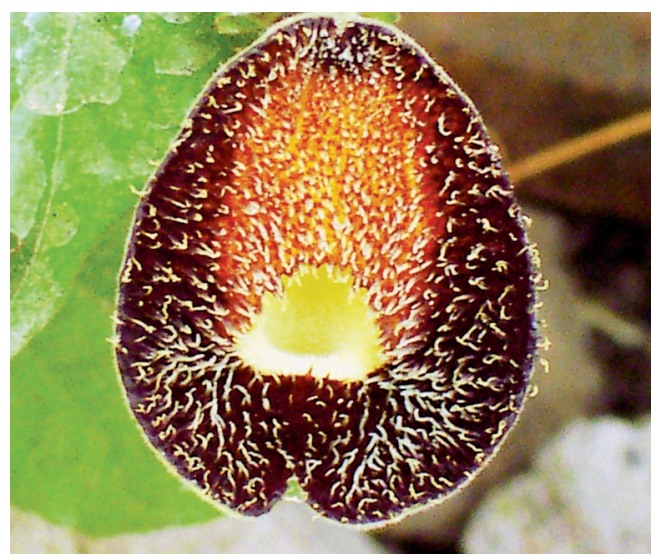

Fig. 2. Aristolochia manantlanensis, detalle del limbo.

4-8 (10) mm de largo y $1 \mathrm{~mm}$ de grueso, bractéolas triangular-cordadas, pilosas, de (4) 5-10 (11) mm de largo, 4-7 (9) mm de ancho; la parte basal del cáliz forma un ángulo de $180^{\circ}$ respecto a la apical, purpúrea, la garganta glabra, amarilla, hipantio no evidente, limbo ovado-cordado, peltado en la antesis, entero, el ápice obtuso, de 2.4$2.8 \mathrm{~cm}$ de largo, 1.7-2.4 cm de ancho, de color púrpura oscuro con una línea verde amarillenta, abundantemente piloso-fimbriado, excepto en la garganta, los tricomas de 0.7-1 mm de largo, tubo tubular con la parte basal adpresa a la apical $\left(180^{\circ}\right)$, de color verde a purpúreo, de (1.7) 1.9-2.2 (2.5) cm de largo, 4-5 mm de diámetro, con tricomas esparcidos, utrículo oblongo-elipsoide, esparcidamente piloso, de 9-11 mm de largo, 5-6 mm de diámetro, siringe excéntrica, tubular, de 2-2.5 mm de largo, 2 $\mathrm{mm}$ de diámetro; ginostemo 5-lobado, subestipitado, de $3 \mathrm{~mm}$ largo, $2 \mathrm{~mm}$ de diámetro, estípite de $1.5 \mathrm{~mm}$ de largo, $1.5 \mathrm{~mm}$ de diámetro, estambres 5 , tetraloculares, anteras de $1.5 \mathrm{~mm}$ de largo, $0.25 \mathrm{~mm}$ de ancho; ovario algo piloso, de 9-11 (12) $\mathrm{mm}$ de largo, 2-2.5 mm de diámetro; fruto capsular, botuliforme, dehiscencia basicida, septífraga, de 2-2.7 cm de largo, 1.5-1.7 cm de diámetro; semillas numerosas, triangulares, de color café obscuro, de 5-6 mm de largo, 5-6 $\mathrm{mm}$ de ancho, $1 \mathrm{~mm}$ de grueso, la superficie ligeramente tuberculada.

Tipo: México, Jalisco, municipio de Cuautitlán de García Barragán, 1 km al NO de San Miguel, 1550 m, vegetación secundaria en medio del cultivo de maíz y Zea diploperennis, 30 de octubre de 2003, F. J. Santana-Michel y D. Zizumbo Villarreal 11593 (holótipo en ZEA; isótipos por distribuirse a los herbarios ENCB, IBUG, IEB y MEXU). 
Material adicional examinado: México, Jalisco, municipio de Cuautitlán de García Barragán, 1 km al SE de San Miguel, camino Ayotitlán-San Miguel, Sierra de Manantlán, 16-18 km NEE de Cuautitlán de García Barragán, 1550 de altitud, vegetación secundaria: Viguiera, Zea diploperennis, Acacia, Senna, 24 noviembre 1988, F. J. Santana-Michel $4246 a$ (en fruto, ZEA); 11805 (ZEA). Jalisco, municipio de Cuautitlán de García Barragán, Peña Blanca, 2 km al SE de San Miguel, Sierra de Manantlán, $1540 \mathrm{~m}$ de altitud, vegetación secundaria: Zea diploperennis, Viguiera, Verbesina, Paspalum, 14 septiembre 2004, F. J. Santana-Michel 11804 (ZEA). Jalisco, municipio de Cuautitlán de García Barragán, La Ventana, 2 km a NO de San Miguel, Sierra de Manantlán, 1740 m de altitud, vegetación secundaria: Zea diploperennis, Viguiera, Verbesina, Hyptis, Senecio, 14 septiembre 2004, F. J. Santana-Michel 11806 (ZEA).

Distribución conocida: México, estado de Jalisco, Sierra de Manantlán, sólo se registra de los alrededores de San Miguel en el municipio de Cuautitlán.

Época de floración: de septiembre a noviembre y quizás hasta diciembre.

Hábitat: cultivo de maíz con Zea diploperennis, vegetación secundaria en campos de cultivo de maíz abandonados donde convive con las siguientes especies: Viguiera ensifolia, Verbesina sphaerocephala, Phaseolus vulgaris, Ipomoea purpurea, Buddleia parviflora, B. sessiliflora, Cirsium anartiolepis, Senecio salignus, Tithonia rotundifolia, Trigonospermum melampodioides, Paspalum candidum, P. squamulatum, Melampodium perfoliatum, Setaria geniculata, S. scandens, Melampodium perfoliatum, Crotalaria longirostrata, Acacia angustissima, Desmodium skinneri var. Alavovirens, Triumfetta semitriloba, Rhodosciadium pringlei.

Siguiendo la disposición de Pfeifer (1970), Aristolochia manantlanensis se ubica en la proximidad de $A$. acontophylla Pfeifer, A. karwinskii Duchr. y A. variifolia Duchr., especies con flores geniculadas y cálices maduros mayores de $3 \mathrm{~cm}$ de largo, pedúnculos cortos y el tubo del cáliz doblado. El cuadro 1 resume las diferencias entre estas plantas.

Las cuatro especies mencionadas se pueden separar de acuerdo con el trabajo de Pfeifer (1970) mediante la siguiente clave:

1. Hojas angostas, sublineares, mucho más largas que anchas, la base con lóbulos pequeños hastados; limbo del cáliz de $3 \mathrm{~cm}$ de ancho, con pequeños tubérculos A. acontophylla 
1. Hojas cerca de una a cuatro veces más largas que anchas; limbo del cáliz cortamente fimbriado a glabrescente.

2. Superficie del limbo del cáliz glabrescente, garganta con la superficie periférica más oscura A. karwinskii

2. Superficie del limbo del cáliz cortamente fimbriada o pilosa.

3. Limbo del cáliz de $2.5 \mathrm{~cm}$ de largo, $1.5 \mathrm{~cm}$ de ancho, ápice del limbo agudo; tubo agrandándose hacia el limbo A. variifolia

3. Limbo del cáliz de $2.4-2.8 \mathrm{~cm}$ de largo, $1.7-2.4 \mathrm{~cm}$ de ancho, ápice del limbo obtuso; tubo tubular A. manantlanensis

Cuadro 1. Comparación de caracteres de A. manantlanensis con otras especies relacionadas.

\begin{tabular}{|c|c|c|c|c|}
\hline & A. acontophylla & A. karwinskii & A. variifolia & A. manantlanensis \\
\hline $\begin{array}{l}\text { Lámina } \\
\text { foliar }\end{array}$ & $\begin{array}{l}\text { linear a } \\
\text { angostamente } \\
\text { lanceolada }\end{array}$ & $\begin{array}{l}\text { triangular-cordada } \\
\text { a hastada } \\
\text { subtrilobada }\end{array}$ & $\begin{array}{l}\text { cordada a } \\
\text { hastada } \\
\text { subtrilobada }\end{array}$ & $\begin{array}{l}\text { sagitada, con la } \\
\text { base bilobada }\end{array}$ \\
\hline Pecíolo & 4-10 $\mathrm{mm}$ de largo & $1-3 \mathrm{~cm}$ de largo & $1-2 \mathrm{~cm}$ de largo & $2-5 \mathrm{~cm}$ de largo \\
\hline Pedúnculo & $1-1.5 \mathrm{~cm}$ de largo & $1-1.5 \mathrm{~cm}$ de largo & $\begin{array}{l}1.2-1.5 \mathrm{~cm} \mathrm{de} \\
\text { largo }\end{array}$ & $4-8 \mathrm{~mm}$ de largo \\
\hline $\begin{array}{l}\text { Limbo del } \\
\text { cáliz } \\
\text { Forma }\end{array}$ & $\begin{array}{l}\text { peltado-orbiculada } \\
\text { café púrpura }\end{array}$ & $\begin{array}{l}\text { ovada, aguda en el } \\
\text { ápice } \\
\text { púrpura oscuro }\end{array}$ & $\begin{array}{l}\text { ovado-cordada, } \\
\text { aguda en el } \\
\text { ápice } \\
\text { café }\end{array}$ & $\begin{array}{l}\text { ovado-cordada, } \\
\text { obtusa en el ápice } \\
\text { púrpura oscuro }\end{array}$ \\
\hline Tubo & tubular & tubular & $\begin{array}{l}\text { no tubular, } \\
\text { agrandándose } \\
\text { hacia el limbo }\end{array}$ & tubular \\
\hline Utrículo & obovado & obcónico & elipsoide & elipsoide \\
\hline
\end{tabular}

Aristolochia manantlanensis se puede identificar fácilmente por sus pedúnculos cortos (4-8 mm), el tubo tubular y el limbo del cáliz obtuso en el ápice.

\section{AGRADECIMIENTOS}

El autor desea agradecer a las autoridades del Centro Universitario de la Costa Sur de la Universidad de Guadalajara y especialmente al Departamento de Ecolo- 
gía y Recursos Naturales y a la Facultad de Ciencias Biológicas de la Universidad de Colima la ayuda obtenida para la realización de este trabajo. También se agradece al Consejo Nacional de Ciencia y Tecnología el apoyo recibido como becario número 125840. A Roberto González Tamayo se le reconoce su colaboración en la revisión del manuscrito. Asimismo doy gracias a los compañeros y colaboradores en el inventario de la región Costa Sur de Jalisco, Ramón Cuevas G., Luis Guzmán H., Nora Minerva Núñez L. y Enrique V. Sánchez Rodríguez. Finalmente quiero agradecer a los revisores anónimos por sus importantes y atinadas sugerencias.

\section{LITERATURA CITADA}

Iltis, H. H., J. F. Doebley, R. Guzmán M. y B. Pazy. 1979. Zea diploperennis (Gramineae): a new teosinte from México. Science 203: 186-188.

Pfeifer, H. W. 1966. Revision of the North and Central American hexandrous species of Aristolochia (Aristolochiaceae). Ann. Missouri Bot. Gard. 53: 115-196.

Pfeifer, H. W. 1970. A revision of the pentandrous species of Aristolochia. The University of Connecticut Publication Series. Storrs, Connecticut. 134 pp.

Santana-Michel, F. J. 1995. Una especie nueva de Aristolochia (subsección Pentandrae) del estado de Jalisco, México. Boletín IBUG 3(1-3): 87-90.

Vázquez, J. A., R. Cuevas, T. Cochrane, H. H. Iltis, F. J. Santana y L. Guzmán. 1995. Flora de Manantlán. Sida Botanical Miscellany 13: 1-312. 